\title{
Haemorrhage in the course of subcardial gastrointestinal stromal tumor - a case description
}

\author{
Tomasz Pedowski ${ }^{1}$, Marlena Pedowska², Wioletta Masiak ${ }^{1}$, Aleksander Ciechański ${ }^{1}$, Grzegorz Ćwik ${ }^{1}$, \\ Andrzej Dąbrowski ${ }^{1}$, Grzegorz Wallner ${ }^{1}$ \\ 1Department and Clinic of General Surgery, Gastroenterology and Digestive Tract Neoplasm, Medical University of Lublin, Poland \\ ${ }^{2}$ Oral Medicine Independent Unit, Medical University of Lublin, Poland
}

Prz Gastroenterol 2014; 9 (1): 53-56

DOI: $10.5114 / p g .2014 .40852$

Key words: oesophageal diseases, gastrointestinal stromal tumor, oesophageal tumor, haemorrhage from gastrointestinal tract.

Address for correspondence: Tomasz Pedowski MD, Department and Clinic of General Surgery, Gastroenterology and Digestive Tract Neoplasm, Medical University of Lublin, 16 Staszica St, 20-081 Lublin, Poland, phone: +48 81523 41 27, e-mail: tomaszpedowski@gmail.com

\begin{abstract}
This paper is a discussion of the case of a 51-year-old female patient admitted urgently for haemorrhage from the upper gastrointestinal tract and increased dysphagia. After preliminary treatment, the patient was qualified for surgery, during which a large lesion was discovered in the shape of a stromal tumor closely connected with the oesophago-gastric junction. A resection of the tumor and part of the oesophagus was carried out, and a cervical fistula was formed. After a 4-month period, the continuity of the gastrointestinal tract was restored by forming a substitute organ out of the ascending colon and the end of the small intestine, anastomosed on the cervix. The patient was discharged in good overall condition.
\end{abstract}

\section{Introduction}

The term gastrointestinal stromal tumor (GIST) is used with reference to a rare malignant neoplasm of the gastrointestinal tract of mesenchymal origin. This type of neoplasm usually occurs in the wall of the gastrointestinal tract and does not normally expand into its lumen but grows in a centrifugal direction.

Stromal neoplasm of the gastrointestinal tract may occur in any of its parts. Occasionally, such tumors are found in the omentum or mesentery and are not linked to the wall of the gastrointestinal tract [1, 2]. Tumors of the GIST type are mostly located in the stomach wall $(60-70 \%)$ and less often in the small intestine wall (20-30\%), in the oesophagus or large intestine wall $(<10 \%)[1,3]$.

In the wall of the gastrointestinal tract, mesenchymal neoplasm may develop in a subserous, intrawall or submucous location. Clinical symptoms and ailments related to the presence of a tumor largely depend on its location and growth direction. Tumors with an outward pattern of growth develop unnoticed, which is why they are not discovered in the early stages. The ailments which accompany them (pain, a feeling of tightness) appear relatively late, which restricts the chances for early detection of the disease and accounts for the high degree of its advancement when diagnosed, often in the form of a palpable lump in the abdominal cavity [1]. Stromal neoplasm in the oesophagus most often manifests itself by dysphagia. Submucous location of the tumor may lead to ulceration, which is often the cause of massive bleeding from the gastrointestinal tract [4-6].

It is known from the literature on the subject that the incidence of GIST is 3 to 20 cases per million inhabitants a year $[2,6,7]$. In $20-30 \%$ of the cases it is in the form of a malignant neoplasm. Estimates for Poland amount to 700-1200 GIST cases a year, of which 140-400 are malignant cases [2]. The GIST metastases may occur as late as 10-15 years after the primary tumor was diagnosed [1, 3, 8].

This paper discusses the case of a patient in whom GIST of the oesophago-gastric junction caused massive bleeding from the upper gastrointestinal tract. Despite repeated attempts at endoscopic treatment, the bleeding turned out to require surgical treatment. The case has been described due to the rare location of the tumor and the method of treatment. 


\section{Case report}

The patient - female, aged 61 years was urgently admitted to the $2^{\text {nd }}$ Department and Clinic of General Surgery, Gastroenterology and Digestive Tract Neoplasm in Independent State Hospital no 1 in Lublin on $6^{\text {th }}$ October 2009 for bleeding from the upper gastrointestinal tract, manifesting itself as tarry stools over the previous 4 days. The patient also felt general weakness and pain in the epigastrium. She had been treated for type 2 diabetes, ischaemic heart disease and persistent atrial fibrillation.

On arrival at the clinic, the patient's general condition was relatively serious. The physical examination revealed paleness of the sweat-covered skin and tarry stools in the rectal ampulla. The ailments were accompanied by hypotonia $(90 / 70 \mathrm{~mm} \mathrm{Hg})$ and tachycardia (100/min). The abdomen did not show pathological resistance and was slightly tender in the epigastrium. In blood cell count determination the number of erythrocytes (RBC) was 2.92 million, the concentration of haemoglobin was $6.2 \%(6.2 \mathrm{~g} / \mathrm{dl})$ and the haematocrit (HCT) value was $21.6 \%$. The patient was given 9 points in risk assessment using the Rockall score.

Medical treatment was applied (intravenous infusion of the inhibitor of the proton pump and drugs activating blood clotting) and 4 units of packed erythrocytes and 2 units of anti-haemophilic plasma were transfused. In gastroscopy, a $5 \mathrm{~cm}$ subcardial tumor was found, covered with mucous membrane with a clot-filled defect on top of the tumor. During the examination argon obliteration was performed on the area around the membrane defect. Initially, the general condition of the patient stabilised (arterial blood pressure and heart action were brought back to normal, the levels of erythrocytes, haematocrit and haemoglobin grew to 3.45 million, $29.3 \%$ and 9.2 mg\%, respectively).

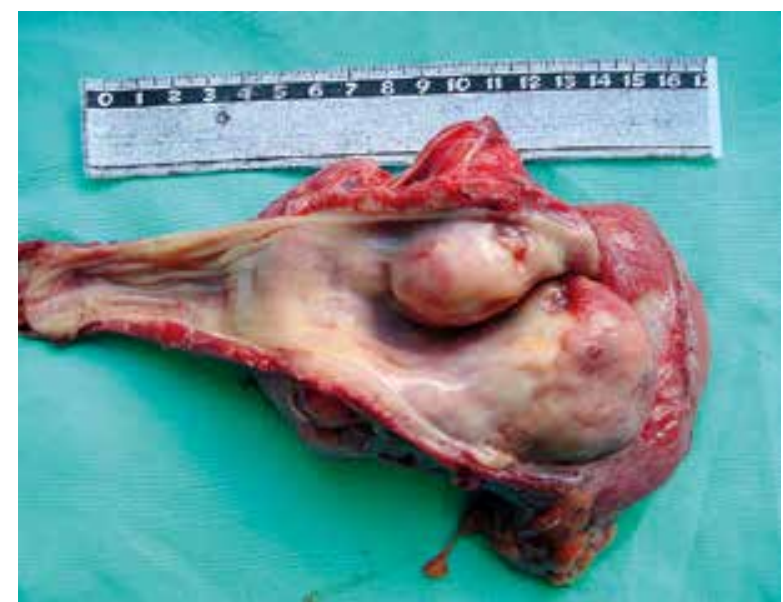

Figure 1. Resected tumor
The day after admission bleeding from the gastrointestinal tract recurred, which was reflected in a decline in morphology, haematocrit, tarry stools and tachycardia. Subsequent medical treatment and another argon obliteration of the tumor surface proved ineffective. On account of persistent bleeding, the patient was qualified for immediate surgery, which began at the following parameter values: 2.72 million erythrocytes, $9.9 \mathrm{mg} \%$ concentration of haemoglobin and $27.7 \%$ of haematocrit. Two units of packed red blood cells were transfused during the operation.

A large irregular tumor (>10 cm) located in the lower back mediastinum and oesophageal hiatus of the diaphragm was found during the operation (Figures 1 and 2). Relatively hard and of irregular shape, the tumor was directly connected with the oesophagus over a $4 \mathrm{~cm}$ section of the oesophago-gastric junction. Although the blood deficit was made up during the surgery, the patient developed symptoms of myocardial ischaemia. Acting on the recommendations of anaesthetists, the duration of the surgery was shortened to reduce the burden on the patient. Esophagectomy with formation of a salivary fistula was performed with access from the abdomen and the left side of the neck. Upper stomach resection was also carried out, and alimentary gastrostomy was installed in the remaining part of the stomach. The overall condition of the patient after the operation was found to be stable. After the morphological parameters levelled off, circulation and respiration stability were restored. Eight days after the surgery the patient was discharged from hospital.

Histopathological assessment of the tumor in the Histopathology Laboratory of Independent Hospital No 1. The following were identified: Neoplasma fusocellulare benignum probabiliter GIST, lymphadenitis reactiva et sinus histiocytosis ( 6 nodes of the lesser curvature

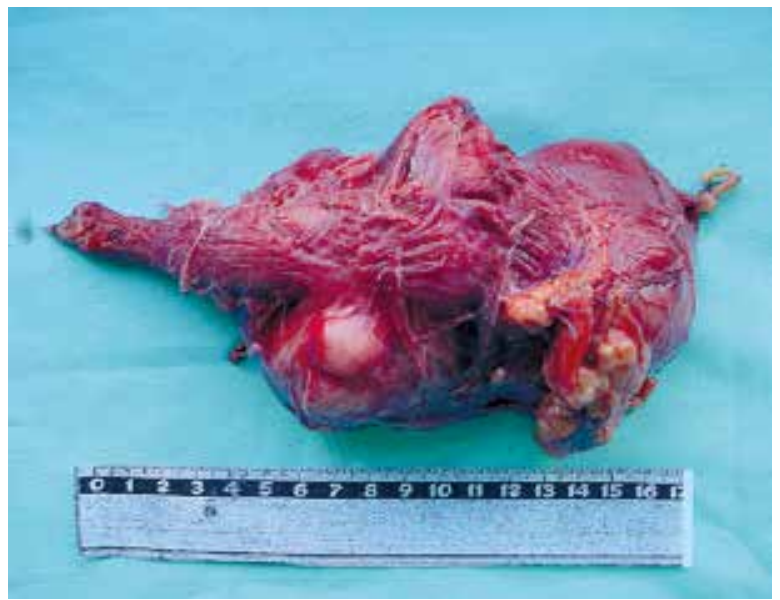

Figure 2. Internal view 
of the stomach). An additional analysis in the Pathology Department of the Oncology Centre Institute in Warsaw confirmed the tumor identification as GIST of low mitotic activity (JM-4/50HPF). An immunohistochemical analysis confirmed the presence of CD117 and CD34 antigens.

Four months later the patient was admitted to the Clinic once more in order to restore the continuity of the gastrointestinal tract. During the operation on $22^{\text {nd }}$ Feb 2010, an oesophageal substitute was formed from the descending colon and the end part of the ileum. After removing the appendix, the intestine was retrosternally shifted to the left half of the neck. The salivary fistula was removed and the proximal part of the oesophagus was anastomosed end-to-side with the distal part of the ileum, while the transverse colon was anastomosed with the front wall of the remainder of the stomach. Gastrostomy was left to ease tension. The proximal part of the ileum was anastomosed with the transverse colon side-to-side. The postoperative course was complicated by the pneumothorax on the right side which entailed draining of the pleural cavity.

During the post-operative period, total parenteral alimentation was implemented, and by gradually making up for the biochemical deficits an improvement in the overall condition of the patient was obtained. On day 7 after the operation, contrast examination of the oesophagus substitute was performed using gastrographin solution (Figures $3 \mathrm{~A}$ and $3 \mathrm{~B}$ ) and a regular passage of the contrast through the anastomoses was observed and no evident craters implying the presence of a fistula were found. The patient began to be fed orally. On day 9 after the operation the gastrostomy was removed, and on day 14 the patient was

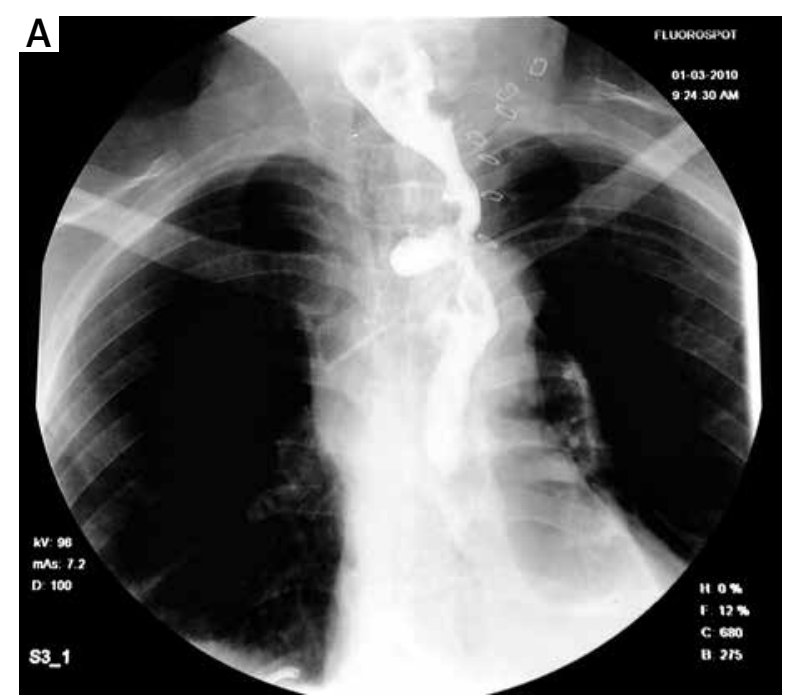

Figure 3. X-Ray postoperative examination discharged from hospital in a good overall condition. A check-up examination 6 months later did not reveal obstacles when receiving solid aliment.

\section{Discussion}

The most frequent causes of upper gastrointestinal bleeding include digestive damage to the duodendum or stomach, oesophageal varices, haemorrhagic inflammation of the mucosa in the stomach and stomach neoplasms $[7,9,10]$. Bleeding from the upper gastrointestinal tract due to a GIST- mesenchymal neoplasm - is one of the rarest causes [2].

Identification of this type of neoplasm is based on immunohistochemical tests for the presence of CD117 and CD34 antigens. CD34, more rarely, is associated with a less aggressive form of the disease. It is assumed that $85 \%$ of GIST tumors show expression of the tyrosine kinase receptor, a protein coded by the KIT $C$ gene. Irregular expression of the extra-epithelial KIT receptor, which is a product of the c-kit proto-oncogene, is made evident by the presence of CD117 antigen. An incorrectly built KIT receptor, formed during the mutation of c-kit proto-oncogene results in considerable proliferative activity of cells $[3,6,9,11]$.

Small tumors of the GIST type located in the upper digestive tract initially do not cause pain and are usually discovered by accident during endoscopic examination. Larger tumors, penetrating into the lumen, may lead to dysphagia, ulceration of the organ wall and bleeding [2, 7]. The source of massive bleeding from the upper digestive tract in the described case turned out to be a large GIST in the oesophago-gastric junction, and its character was confirmed in immune-histochemical tests (presence of CD117 and CD34 antigens).

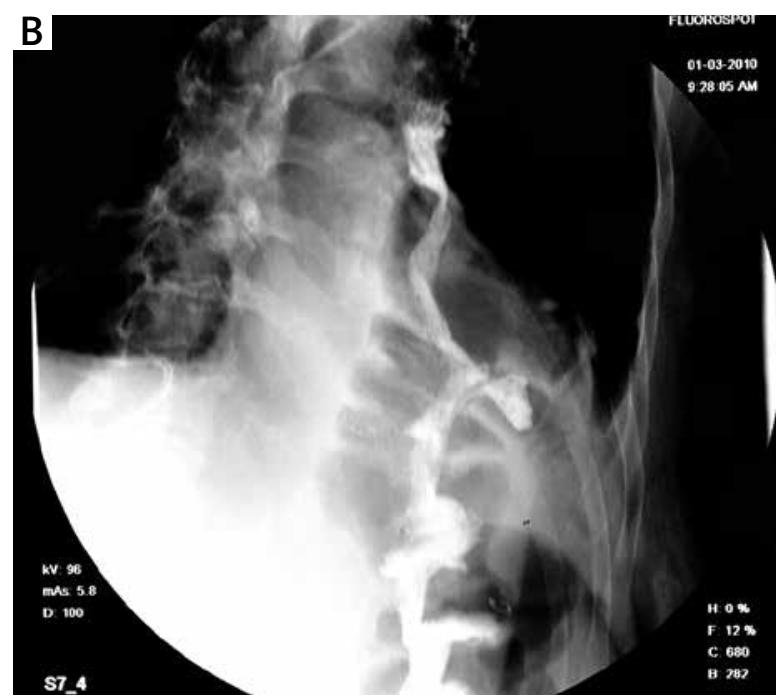


As indicated in the literature of the subject, GISTs of the digestive tract are most frequently located in the stomach $[9,10,12]$. Their surface may be ulcerated, which is the reason for bleeding. These observations by other authors have been validated by the described case. Endoscopic examination revealed a subcardial stomach tumor with a mucosa deficit on top, which was responsible for the bleeding. Attempts at pharmacological treatment and argon ablation of the bleeding spot proved inefficient and the patient was urgently qualified for surgery. During the operation, the size, location and close connection of the tumor with the oesophagus and stomach wall in the oesophago-gastric junction area made it impossible to remove the tumor without a resection of this part of the digestive tract. In the described case, it was particularly difficult to decide on the best method of surgical treatment. At first, a one-step operation was planned, comprising partial resection of the stomach and the lower oesophagus and oesophago-gastrostomy within the chest. The poor general condition of the patient (symptoms of myocardial ischaemia aggravated by blood loss) excluded this type of operation. The situation required a maximum shortening of the operation time. Upper stomach resection with alimentary gastrostomy and esophagectomy without opening the chest were performed, and a salivary fistula was made in the neck. Four months later, continuity of the gastrointestinal tract was restored using the right colon and part of the ileum. The patient took the operation well. An examination carried out 6 months later did not show malfunction when receiving food orally. According to the authors, the choice of treatment tactics was justified. The patient must undergo regular oncologic and surgical check-ups due to the fact that the described tumor, with a diameter greater than $10 \mathrm{~cm}$, is a neoplasm type of uncertain prognosis and is capable of recurring and metastasising, despite its low mitotic activity $[1,3]$.

\section{References}

1. Miettinen M, Majidi M, Lasota J. Pathology and diagnostic criteria of gastrointestinal stromal tumors (GISTs): a review. Eur J Cancer 2002; 38 Suppl. 5: S39-51.

2. Stachura J, Limon J, Ruka W, Karcz D. GISTs - Gastrointestinal stromal tumors. Via Medica, Gdańsk 2002.

3. Miettinen M, Lasota J. Gastrointestinal stromal tumors: pathology and prognosis at different sites. Semin Diagn Pathol 2006; 23: 70-83.

4. Degrate L, Nobili C, Garancini M, et al. Challenging diagnosis of ileal gastrointestinal stromal tumor presenting with obscure digestive bleeding. Tumori 2009; 95: 823-7.

5. Gourgiotis S, Kotoulas D, Aloizos S, et al. Preoperative diagnosis of obscure gastrointestinal bleeding due to a GIST of the jejunum: a case report. Cases J 2009; 2: 9088.
6. Prywiński S, Szopiński J, Wierzchowski P, Dąbrowiecki S. Gastrointestinal stromal tumor of small intestine as the cause of massive gastrointestinal hemorrhage - case report. Pol Surg 2008; 10: 107-12.

7. Brian P, Rubin BP, Heinrich MC, Corless CL. Gastrointestinal stromal tumour. Lancet 2007; 369: 1731-41.

8. Głuszek S, Rylski R, Kot M, et al. GIST - risk of recurrence and dissemination. Prz Gastroenterol 2008; 3: 176-84.

9. Gupta P, Tewari M, Shukla HS. Gastrointestinal stromal tumor. Surg Oncol 2008; 17: 129-38.

10. Raquel E, Davila RE, Faigel DO. GI stromal tumors. Gastrointest Endosc 2003; 58: 80-8.

11. Lewosiuk A, Białek A, Smereczyński A, Starzyńska T. Submucosal tumors of upper gastrointestinal tract. Prz Gastroenterol 2009; 4: 126-36.

12. Zaręba K, Kamocki Z, Hołody-Zaręba J, et al. A rare case of simultaneous presence of adenocarcinoma, gastrointestinal stromal tumour and carcinoid tumour in the stomach. Prz Gastroenterol 2010; 5: 297-300.

Received: $\quad 7.09 .2011$

Accepted: $\quad 12.02 .2012$ 